\title{
The effect of a hysteroscopic niche resection compared with LNG-IUD on postmenstrual spotting in women with a symptomatic niche in the uterine cesarean scar:a prospective cohort study
}

Xiaoqing $\mathrm{He}^{1}$, liang yan ${ }^{1}$, xiaoya zhao ${ }^{1}$, li yan ${ }^{1}$, chuqing $\mathrm{he}^{1}$, xiaofeng wang $^{1}$, Guiling Liang $^{2}$, hang $\mathrm{Qi}^{1}$, Chenfeng Zhu ${ }^{2}$, Ben Mol${ }^{3}$, Jian Zhang ${ }^{4}$, and Judith Huirne ${ }^{5}$

${ }^{1}$ Affiliation not available

${ }^{2}$ Department of Obstetrics and Gynecology, International Peace Maternity and Child Health Hospital, School of Medicine, Shanghai Jiaotong University, Shanghai, China

${ }^{3}$ Monash University Medical Centre

${ }^{4}$ International Peace Maternity and Child Health Hospital, School of Medicine, Shanghai Jiaotong University, Shanghai, China.

${ }^{5}$ Amsterdam UMC - Locatie VUMC

September 11, 2020

\begin{abstract}
Objective: To compare the effect of a hysteroscopic niche resection with a Levenorgestrel-releasing intrauterine device (LNGIUD) on postmenstrual spotting in women with a symptomatic niche in the uterine cesarean scar. Design: Single center, prospective cohort study. Setting: A hospital affiliated to a medical college in China. Population: Women diagnosed with a niche by MRI scan and postmenstrual spotting of at least two days. Method: Women were allocated to two groups based on the shared medical decision-making approach, and were followed up for 1 year after treatment. Main outcome measures: The primary outcome was effectiveness in reducing postmenstrual spotting. Secondary outcomes were menstrual characteristics, direct medical costs, complications and side effects. Results: Effectiveness of LNG-IUD was significantly higher than a hysteroscopic niche resection during the first year, based on linear mixed models $(\mathrm{P}=0.009)$, and the effectiveness increased overtime within 1 year. Postmenstrual spotting reduced statistically in both groups. More slight side effects, although not statistically significant, were reported in LNG-LUD group at obviously lower direct medical costs. Conclusion:LNG-IUD is more effective in the treatment of postmenstrual spotting from the 6th month onwards than a hysteroscopic niche resection in women with a symptomatic niche at lower direct costs. Key Words:Niche, uterine cesarean scar, hysteroscopy niche resection, LNG-IUD, postmenstrual spotting, amenorrhea Tweetable abstract: LNG-IUD is more effective in the treatment of postmenstrual spotting related to a niche than hysteroscopic niche resection.
\end{abstract}

\section{Funding}

This work was supported by Clinical Research Project of Shanghai Health and Fitness Commission (201940012), Shanghai Municipal Key Clinical Specialty (shslczdzk01802), Scientific Research Project of Shanghai Health and Fitness Commission (20184Y0344), International Peace Maternal and Child Health Institute Clinical Research Program (GFY5826), and Medical Engineering Cross Youth Funds from Shanghai Jiao Tong University (YG2017QN38), Translational Medicine Cross Fund from Shanghai Jiao Tong University (ZH2018QNA36).

\section{Introduction}


Cesarean section (CS) is one of the most common performed large surgical intervention in, and its incidence has been increasing steadily, accounting worldwide for one-third of all deliveries. ${ }^{1}$ Only recently we became aware of long-term morbidity related to these CS that may be related to a defect in the uterine CS scar, also called a niche. It is defined as a disruption of the integrity of the myometrium at the site of the cesarean scar with a minimum depth of $2 \mathrm{~mm}$ using (sono)hysterography. ${ }^{2}$ Other diagnostic methods include magnetic resonance imaging (MRI) or hysteroscopy. ${ }^{3-5}$ Niches also called Cesarean scar defects are reported in $49.6 \%$ $\sim 64.5 \%$ in patients with at least one previous CS, and it increases along with the number of CSs up to $100 \%$ women with 3 or more CSs. ${ }^{6-8}$

Niches are associated with gynecological symptoms like postmenstrual spotting, chronic pelvic pain, infertility and complications in subsequent pregnancy. Postmenstrual bleeding is the most common symptom that is reported in 29\%-82\% in women with a niche. It severely affects women's health and quality of life, in particular due to its unpredicted nature..$^{3,9-11}$ In China, more and more women with a diagnosed niche in the uterine scar ask for surgery or medical treatment in order to treat postmenstrual spotting according to the clinical experience.

So far, international acknowledged guidelines on the preferred treatment for this indication are lacking. Nonsurgical and surgical methods have been reported for the treatment of niche related bleeding symptoms. ${ }^{12,}{ }^{13}$ Surgical options include laparoscopic, transvaginal and hysteroscopic niche resections. In women with a relatively small niche with adequate residual myometrial thickness (mostly $>2.5 \mathrm{~mm}$ ), a hysteroscopic niche resection is the least invasive approach. ${ }^{14-16}$ A hysteroscopic niche resection reduces postmenstrual spotting with an average of 4 days. ${ }^{12,17}$ Non-surgical methods include continuous or cyclic hormonal therapies and Levenorgestrel-releasing intrauterine device (LNG-IUD). ${ }^{12}$ Although the number of studies evaluating the effect of these medical treatments in the reduction of postmenstrual spotting is very limited. Concerning LNG-IUD only one study, including 6 patients only, indicated a positive effect. ${ }^{18}$ As far as we are aware of comparative studies between hysteroscopic niche resection and LNG-IUD in the treatment of postmenstrual spotting lacking. Therefore, we conducted a prospective cohort study to compare the effect of a hysteroscopic niche resection and LNG-IUD on niche related postmenstrual spotting.

\section{Methods}

\section{Study Design}

Single center prospective cohort study with consecutive inclusion of patients and structured follow-up every three months during the first year after inclusion.

\section{Population}

Women aged between 20-45 years, diagnosed with a niche by MRI scan with postmenstrual spotting of at least two days who considered to undergo a hysteroscopic niche resection or to receive a LNG-IUD between April 2018 and April 2019 in the International Peace Maternity and Child Health Hospital affiliated to Shanghai Jiao Tong University School of Medicine, China were asked to participate in the study, consecutively. Exclusion criteria were fertility wish within 1year, irregular menstrual cycle before the last CS, the presence of an intrauterine device, coagulopathy, current exogenous hormone treatment, and other gynecological conditions that could cause prolonged bleeding such as leiomyoma, adenomyoma, endometrial hyperplasia, ovarian endometriosis and pregnancy. In the hysteroscopic niche resection group, additional exclusion criteria were contraindication for general anesthesia and a residual myometrial thickness of less than $2 \mathrm{~mm}$.

\section{Procedures}

The type of treatment was based on the shared medical decision-making approach.

LNG-IUD (52mg)

LNG-IUD were inserted according to a standardized protocol in the outpatient department. Patients were in lithotomy position, after bimanual examination of the uterine size and position and cervix disinfection, 
the cervix was grasped with a tenaculum forceps and length of the uterine cavity was assessed and had to be between $6-10 \mathrm{~cm}$. The LNG-IUD was gently inserted after aspiration of the endometrium for histological examination. Correct location of the LNG-IUD was confirmed by transvaginal ultrasound.

Hysteroscopic niche resection

Hysteroscopic niche resection was performed according to a standardized protocol under the general anesthesia in the Daycare Surgery department. A resectoscope (Ch. 26 model WA22061 with a $12^{\circ}$ optic 22001A) equipped with a 3-mm-deep and 5-mm-wide loop (Olympus, Germany) was used. As distention medium we used $0.9 \% \mathrm{NaCl}$ under $120 \mathrm{mmHg}$ intrauterine pressure. Patients were in lithotomy position, after bimanual examination to determine the size and the position of the uterus, the cervix was disinfection and grasped with a tenaculum forceps. Dilatation of the cervix up to Hegar 9, aspiration of the endometrium for histological examination and hysteroscopic examination of the uterine cavity. Then the outflow tract was resected and the niche surface including all small vessels in the niche were coagulated (Figure S1). Patients were observed postoperatively for at least 3 hours.

Both procedures were performed by one trained doctor with extensive experience in performing the procedures.

\section{Outcome measures}

The primary outcome measure was proportion of patients with a reduction of postmenstrual spotting of more than 2 days at each follow-up point and without the need for additional therapy, using the ITT analyses, we called it the effective rate. If an IUD was removed because of symptoms or if additional therapies were applied, we considered this as a failure. We defined postmenstrual spotting as brownish discharge for more than two days at the end of the menstruation, with a total duration (menstruation and spotting) of more than seven days, or intermenstrual blood loss that starts after the end of the menstruation. ${ }^{3}$ Menstrual characteristics were self-recorded in a menstruation diary at 3,6,9 and 12 months after inclusion and uploaded in an electronic data system (Figure S2).

Secondary outcome measures were: menstrual characteristics (eg, duration of menstruation, total days of blood loss per month, presence of spotting, amenorrhea was defined as no menstruation/irregular bleeding or spotting for at least 3 months ${ }^{19}$ and need for treatment because of gynecological complaints); direct medical costs (including the costs of preoperative examination, medical consumption and the surgery); Complications and side effects were evaluated using digital questionaires, these included questions on urinary tract injury, fever, infection, breast pain, pelvic pain, emotional wings, depression, weight changes and acne;

MRI measurements at baseline were: presence of a niche (depth of [?] 2mm), width, depth, length and thickness of residual myometrium (TRM), uterine position. Measurements were performed in line with previous studies, ${ }^{17,20}$ and as recommended by a group of international experts (see Figure S3). ${ }^{21}$

\section{Statistical analysis}

We calculated sample-size by two independent proportions. ${ }^{17,}{ }^{18}$ Using estimated 6-month improvement rate of $83 \%$ with LNG-IUD insertion and $71 \%$ with hysteroscopic niche resection based on our pilot study, and assuming an alpha level of 0.025 for a one-sided test, we calculated that a sample of 68 cases who underwent therapy would result in $80 \%$ power (1:1 ratio). The sample size was increased to 76 to allow for a $10 \%$ rate of loss to follow-up over the 12-month period.

All statistical tests were conducted using IBM SPSS 23(SPSS Inc., Chicago, IL, USA). We used both ITT and per protocol analyses. For the primary outcome (effective rate)was based on the ITT analyses. The mean +- standard deviation was used to expressed the continuous data that were normally distributed, the differences between groups were tested using the student $t$ test. Otherwise median and interquartile range (IQR) were used and tested with Mann-Whitney U test. Categorical variables were compared by the $\chi^{2}$ test; if more than $25 \%$ of cells were found to have an expected value of between 1 and 5 , Continuity Correction Chi-Square test was used instead; if more than $25 \%$ of cells were found to have an expected 
value of less than 1, Fisher's exact test was used. The mix linear model was used to compare the effective rate of spotting symptoms repeatedly measured within 1 year after surgery between two groups and the spotting days between each follow-up time points and baseline. Linear-by-linear association test was used to calculated the $\mathrm{P}$ for trend to expressed the efficacy change over time. Logistic regression analysis was used to analyze the effect of the TRM and also of age on spotting and total bleeding days at each follow-up point. The Statistical significance was determined by a $\mathrm{p}$ value of $<0.05$.

\section{Results}

\section{Participants}

As shown in the flow chart (Figure 1), 78 out of the 82 eligible patients gave informed consent and were included. Patients were divided into 2 groups according to their treatment of choice, 40 patients received insertion of LNG-IUD and 38 patients underwent a hysteroscopic niche resection. In the LNG-IUD group, 2 patients were excluded because of endometrial hyperplasia that was diagnosed postoperatively, 2 patients removed the LNG-IUD because of fertility desire, 36 patients finally completed the one-year follow-up and were included in the ITT analyses. Among these 36 patients, 1 patient removed the LNG-IUD for pelvic pain in the $2^{\text {nd }}$ month, 1 patient removed the LNG-IUD for the partial expulsion in the $7^{\text {th }}$ month. In the hysteroscopy group, 2 patients received LNG-IUD because of persisting bleeding symptoms in the $3.5^{\text {th }}$ and $8^{\text {th }}$ month respectively; 2 patients had an unintended pregnancy in the $7^{\text {th }}, 8^{\text {th }}$ month respectively, and then decided to continue the pregnancy; all 38 patients in the hysteroscopy group completed the one-year follow-up and were included in the ITT analyses.

\section{Baseline characteristics}

Baseline characteristics were comparable in both groups, apart from the TRM that was smaller in the LNGIUD group, and there was a trend for higher younger age in the LNG-IUD group (see table1). TRM was not of influence on improvement of postmenstrual spotting on any of the follow-up point during the first year (in the $3^{\text {rd }}$ month: $\mathrm{P}=0.706$; the $6^{\text {th }}$ month: $\mathrm{P}=0.338$; the $9^{\text {th }}$ month: $\mathrm{P}=0.099$; the $12^{\text {th }}$ month: $\mathrm{P}=0.081$ ). The influence of age on improvement of postmenstrual spotting on any of the follow-up point during the first year (in the $3^{\text {rd }}$ month: $\mathrm{P}=0.015$; the $6^{\text {th }}$ month: $\mathrm{P}=0.131$; the $9^{\text {th }}$ month: $\mathrm{P}=0.123$; the $12^{\text {th }}$ month: $\mathrm{P}=0.102)$.

\section{Primary Outcomes}

Both treatments reduced postmenstrual spotting statistically significantly compared to baseline $(\mathrm{P}<0.001$, see table 2). Median spotting reduced from 7 days at baseline to 2 days after hysteroscopic niche resection at $3^{\text {th }}, 6^{\text {th }}, 9^{\text {th }}$ and $12^{\text {th }}$ month, while after LNG-IUD it reduced to 2 days at $3^{\text {th }}$ month follow-up to 0 day from $6^{\text {th }}$ month onwards (see table 3 ). Comparing postmenstrual spotting during the first year, using linear mixed model on ITT analyses base, LNG-IUD was more effective in reducing postmenstrual spotting than the hysteroscopic niche resection ( $\mathrm{p}=0.009$, see table 2$)$.

Total number of women with more than 2 days reduction in spotting at one-year follow-up without removal of the IUD because of symptoms and without additional applied therapies (the effective rate) was 33 out of $36(91.7 \%)$ in the LNG-IUD group and 28 out of 38 (73.7\%) in the hysteroscopic niche resection group $(\mathrm{p}=0.042)$. The effective rate increased over time within 1 year after the insertion of LNG-IUD (66.7\%, $86.1 \%, 91.7 \%, 91.7 \%$, p for trend=0.003), while no trend change was observed in hysteroscopy group $(76.3 \%$, $73.7 \%, 71.1 \%, 73.7 \%$, p for trend=0.743) (see figure S4). Also the reduction in total bleeding days per month including postmenstrual spotting was statistically significantly stronger in the LNG-IUD group from 6 months onwards (see table 3).

In the LNG-IUD group, the rate of amenorrhea increased during the first year from $28 \%$ at 3 months to $39 \%$ at 12 months follow-up. And irregular bleeding reduced from $23 \%$ at 3 months to $3 \%$ at 12 months follow-up. Despite the good results, 11 out of 36 (31\%) patients in the LNG-IUD group were concerned about their ovarian function because of the reduction in menstrual bleeding and requested for removal of the 
LNG-IUD. However, after repeated one-by-one health education about the function of the LNG-IUD all of these patients came back on their request and kept the LNG-IUD.

In the hysteroscopic niche resection group, 2 patients who got pregnant at the $7^{\text {th }}$ and $8^{\text {th }}$ month were amenorrhoic, all others had regular menstrual cycles at 12 months follow-up.

We also conducted the per protocol analyses, 2 patients removed their IUD in the LNG-IUD group and 2 patients got pregnant in the hysteroscopy group and 2 received an additional IUD in the hysteroscopy group and were excluded at the corresponding time points respectively; 34 patients finally finished the one-year follow-up based on the per protocol analyses in both groups (see table S1). The results at each follow up points were comparable with the results of ITT analyses.

\section{Reported complications or side effects}

There were no complications or side effects reported in the hysteroscopic niche resection group ( 0 out of 38 $(0.0 \%)$ ) while 5 out of $36(13.9 \%)$ were reported in the LNG-IUD group, see table $\mathrm{S} 2, \mathrm{p}=0.103)$. There was no bladder injury, fever, urinary tract infection nor where there any signs of acne or depression reported in both groups. In the hysteroscopic niche resection group no side effects were reported. In the LNG-IUD group 2 patients reported weight gain of more than 5 kilograms and breast distended pain, in 2 patients partial IUD expulsion was reported and in 1 patient the LNG-IUD was removed because of pelvic pain.

In the hysteroscopy group, 2 patients received additional treatment (LNG-IUD insertion) because of persisting postmenstrual spotting without improvement. While in the LNG-IUD group, as reported above the LNG IUD was removed in 2 patients because of side-effects.

The mean direct medical costs were 5650[5428,5923] CNY in the hysteroscopy group and 1683[1653,1763] CNY in the LNG-IUD group, pi0.001). This in correspond with $817[785,856]$ \$ and $243[239,255]$ \$ respectively.

\section{Discussion}

Our study demonstrates that both the application of LNG-IUD and hysteroscopic niche resection are effective in reducing postmenstrual spotting and total duration of bleeding per month during the first year. However, from 6 months onwards LNG-IUD was more effective in reducing spotting and total menstrual bleeding days than the hysteroscopic niche resection. The effectiveness of LNG-IUD in reducing spotting increased during the first year while the effect of the hysteroscopic niche resection remained stable. From 6 months onwards reduction in postmenstrual spotting was 8 days in the LNG-IUD group and it was 5 days form 3 months onwards in the hysteroscopic niche resection group. Minor side effects were only reported in the LNG-IUD group. From a medical cost-effective perspective is the LNG-IUD superior to a hysteroscopic niche resection. Additionally, a LNG-IUD can be placed in an outpatient department without any delay and therefor gives less pressure on clinical capacity and facilities.

\section{Comparison to other literature}

The reduction effect of hysteroscopic niche resection on spotting in our study are in line with previous studies reporting a median reduction of 4 days at 3 to 6 months follow-up. ${ }^{11,12,17}$ Only one small study including only 6 patients evaluated the effect of LNG-IUD on spotting and reported a beneficial effect. ${ }^{18}$ None of the previous studies compared both interventions and none of these studies evaluated the changes in effectiveness over time in this population.

In a general population approximately $20 \%$ of amenorrhea is reported one year after insertion of LNGIUD. ${ }^{22-25}$ In our population of regular menstruating patients with spotting and a niche, the rate of amenorrhea was higher, $39 \%$ at one-year follow up. Previous studies also reported higher rates, up to $25 \%$ of irregular bleeding 6 months after LNG-IUD insertion ${ }^{26}$, while only $11 \%$ or our patients reported irregular bleeding in the LNG-IUD group.

The strict clear selection criteria may have played a role in the reported differences between our study and 
previous studies, since women with an irregular menstrual cycle or endometrial atypia were exclusion criteria in our study. This is in line with the fact that previous studies have found in inverse relation between the thickness of the endometrium and the amenorrhoic rate. ${ }^{19,} 27$ In our study the proportion of women with amenorrhea increased over time, this is also in line with previous studies. ${ }^{19,} 27$

\section{Strength and limitations}

As far as we are aware of is this the first study comparing the effect of a hysteroscopic niche resection with LNG-IUD insertion on the reduction of spotting in women with a niche in the CS scar. The strength of our study is that we evaluated symptoms and side effects in a prospective and structured way using menstrual diaries. We had a homogeneous population due to the clear inclusion and exclusion criteria. Consecutive inclusion of patients, structured follow-up at four fixed evaluation points and the very low drop-out rate in both study groups reduce the risk on selection and verification bias.

However, our study has also some limitations. First, this study was divided into groups based on sharedmedical decisions making this increases the risk on selection bias in comparison to a randomized design. This is underlined by the differences in age and in TRM between the two groups. Although additional analyses have shown that these variables were not related to the primary outcome. Due to the small sample size we were not able to study the effect of other potential confounders. Future randomized controlled studies of sufficient sample size should be carried out in the future to confirm our data.

\section{Clinical implications}

Both therapies have proven to be effective in the reduction of spotting in

women with postmenstrual spotting of at least 2 days and a niche in the uterine CS scar, without signs of endometrial atypia and without a desire to become pregnant within one year. However, the LNG-IUD is superior from 6 months onwards in the reduction of spotting and with substantially lower direct medical costs. In women without a wish to conceive the LNG-IUD has the additional advantage that is also has a contraceptive effect. Based on our data LNG-IUD should be offered in this population as a first line treatment. However, LNG-IUD is not an option in women with an active desire to become pregnant.

After LNG-IUD insertion, the effect improves in time during the first year. It is important to provide this information to patients before insertion to prevent premature removal of the LNG-IUD or unneeded additional therapies during the first months after insertion. In the hysteroscopic niche resection group the optimal effect is already achieved after 3 months. Our results indicate that if spotting does not improve substantially at three months after hysteroscopic niche resection, there is no need to wait much longer before considering additional treatments. In our study 2 patients received an additional LNG-IUD with good results.

Another advantage of the LNG-IUD is that its application is independent of the TRM, while a hysteroscopoic niche resection in general is applied in women with a TRM > than $2.5 \mathrm{~mm}$ in order to prevent any bladder injury. ${ }^{11,16,28-30}$ The additional advantage of an LNG-IUD over a hysteroscopic niche resection is that it is easy to insert, does not require surgical instruments or operating room facilities. Hysteroscopic correction operation demands a longer learning curve for the surgeons, and its' safety and efficacy are more likely to be affected by the surgeon's proficiency. Moreover, it is in generally performed under general anesthesia requiring a longer recovery.

Also, even after clear instructions and counseling of our patients almost $1 / 3$ of the patients in the LNG-IUD group were concerned about their ovarian function due to the change of their menstrual pattern and requested initially for removal of their LNG-IUD. This removal could be prevented by repeated health education. This underlines the importance of clear and repeated health education in women considering a LNG-IUD in order to improve compliance and to reduce negative experiences or thoughts.

\section{Conclusion}


The application of LNG-IUD is superior to hysteroscopic niche resection in the treatment of postmenstrual spotting in women with niche in the uterine CS scar and with lower direct medical costs. Therefore, this therapy should be offered as a first line therapy in women with a niche and postmenstrual spotting without the desire to conceive within one year. The effect of LNG-IUD increased over time during the first year while the optimal effect of a hysteroscopic niche resection is reached after 3 months. Proper counseling of patients receiving LNG-IUD prevents unneeded premature removal of the IUD.

\section{Abbreviation}

LNG-IUD: Levenorgestrel-releasing intrauterine device

IUD: Intrauterine device

MRI: Magnetic resonance image

ITT: Intention to treat

CNY: Chinese yuan

\section{Disclosure of interest}

We declare that we have no financial and personal relationships with other people or organizations that can inappropriately influence our work, there is no professional or other personal interest of any nature or kind in any product, service and/or company that could be construed as influencing the position presented in, or the review of, the manuscript entitled.

\section{Authors' contributions}

Jian Zhang and JAF Hurine conceived and designed this study; Jian Zhang, JAF Hurine and Ben W. Mol contributed to the manuscript editing and language editing; Xiaoqing He was responsible for the data analysis and manuscript writing; Yan Liang, Xiaoya zhao, Li Yan, Chuqing He, Xiaofeng Wang, Guiling Liang, Hang Qi and Chenfeng Zhu contributed to the data collection. The final version of manuscript has been approved by all authors.

\section{Details of ethics approval}

All patients provided informed consent, and all data have been anonymized. This study was approved by the Ethics Committee of the institutional ethics committee of the International Peace Maternity and Child Health Hospital in Shanghai, China (GKW 2018-42).

\section{Acknowledgements}

We appreciated all the participants in this study and all the staff at International Peace Maternal and Child Health Hospital for their precise data recording.

\section{Reference}

1.Menacker F, Declercq E, Macdorman MFJSiP. Cesarean Delivery: Background, Trends, and Epidemiology. 2006;30(5):235-41.

2.Antila-Långsjö R, Mäenpää JU, Huhtala H, Tomás E, Staff S. Comparison of transvaginal ultrasound and saline contrast sonohysterography in evaluation of cesarean scar defect: a prospective cohort study. 2018;97(9):1130-6.

3.Bij de Vaate AJM, Brölmann HAM, van der Voet LF, van der Slikke JW, Veersema S, Huirne JAF. Ultrasound evaluation of the Cesarean scar: relation between a niche and postmenstrual spotting. 2011;37(1):93-9.

4.Yao M, Wang W, Zhou J, Sun M, Zhu J, Chen P, et al. Cesarean section scar diverticulum evaluation by saline contrast-enhanced magnetic resonance imaging: The relationship between variable parameters and longer menstrual bleeding. 2017;43(4):696-704. 
5.Wong WSF, Fung WT. Magnetic Resonance Imaging in the Evaluation of Cesarean Scar Defect. Gynecol Minim Invasive Ther. 2018;7(3):104-7.

6.Vikhareva Osser O, Jokubkiene L, Valentin L. High prevalence of defects in Cesarean section scars at transvaginal ultrasound examination. Ultrasound Obstet Gynecol. 2009;34(1):90-7.

7.Wang C-B, Chiu W-W-C, Lee C-Y, Sun Y-L, Lin Y-H, Tseng C-J. Cesarean scar defect: correlation between Cesarean section number, defect size, clinical symptoms and uterine position. 2009;34(1):85-9.

8.van der Voet L, Bij de Vaate A, Veersema S, Brölmann H, Huirne J. Long-term complications of caesarean section. The niche in the scar: a prospective cohort study on niche prevalence and its relation to abnormal uterine bleeding. 2014;121(2):236-44.

9.Borges LM, Scapinelli A, Depes DDB, Lippi UG, Lopes RGCJJoMIG. Findings in Patients with Postmenstrual Spotting with Prior Cesarean Section. 2010;17(3):361-4.

10.Reiter M, Schwope R. Finding a niche: Magnetic resonance imaging located an often-overlooked source of uterine bleeding. American Journal of Obstetrics \& Gynecology. 2014;210(2):171.e1-.e2.

11.Vervoort AJMW, Van der Voet LF, Witmer M, Thurkow AL, Radder CM, van Kesteren PJM, et al. The HysNiche trial: hysteroscopic resection of uterine caesarean scar defect (niche) in patients with abnormal bleeding, a randomised controlled trial. 2015;15(1):103.

12.van der Voet LF, Vervoort AJ, Veersema S, BijdeVaate AJ, Brölmann HA, Huirne JA. Minimally invasive therapy for gynaecological symptoms related to a niche in the caesarean scar: a systematic review. BJOG : an international journal of obstetrics and gynaecology. 2014;121(2):145-56.

13.Stegwee SI, Beij A, de Leeuw RA, Mokkink LB, van der Voet LF, Huirne JAF. Niche-related outcomes after caesarean section and quality of life: a focus group study and review of literature. Quality of life research : an international journal of quality of life aspects of treatment, care and rehabilitation. 2020;29(4):1013-25.

14.Xie H, Wu Y, Yu F, He M, Cao M, Yao SJG, et al. A Comparison of Vaginal Surgery and Operative Hysteroscopy for the Treatment of Cesarean-Induced Isthmocele: A Retrospective Review. 2014;77(2):78-83.

15.Brown K, Tkacz ZJJoO, Gynaecology. Hysteroscopic and laparoscopic management of caesarean scar (niche) defects in symptomatic patients. 2018;38(5):730-.

16.Vitale SG, Ludwin A, Vilos GA, Torok P, Tesarik J, Vitagliano A, et al. From hysteroscopy to laparoendoscopic surgery: what is the best surgical approach for symptomatic isthmocele? A systematic review and meta-analysis. 2020;301(1):33-52.

17.Vervoort A, van der Voet L, Hehenkamp W, Thurkow A, van Kesteren P, Quartero H, et al. Hysteroscopic resection of a uterine caesarean scar defect (niche) in women with postmenstrual spotting: a randomised controlled trial. 2018;125(3):326-34.

18.Chen YY, Tsai CC, Lan KC, Ou YC. Preliminary report on the use of a levonorgestrel intrauterine system for the treatment of intermenstrual bleeding due to previous cesarean delivery scar defect. The journal of obstetrics and gynaecology research. 2019;45(10):2015-20.

19.Mejia M, Mcnicholas C, Madden T, Peipert JFJC. Association of baseline bleeding pattern on amenorrhea with levonorgestrel intrauterine system use. 2016;94(5):556-60.

20.Glavind J, Madsen LD, Uldbjerg N, Dueholm M. Cesarean section scar measurements in non-pregnant women using three-dimensional ultrasound: a repeatability study. European Journal of Obstetrics \& Gynecology and Reproductive Biology. 2016;201:65-9.

21.Jordans IPM, de Leeuw RA, Stegwee SI, Amso NN, Barri-Soldevila PN, van den Bosch T, et al. Sonographic examination of uterine niche in non-pregnant women: a modified Delphi procedure. Ultrasound Obstet Gynecol. 2019;53(1):107-15. 
22.Rozenberg P, Goffinet F, Philippe HJ, Nisand I. Ultrasonographic measurement of lower uterine segment to assess risk of defects of scarred uterus. The Lancet. 1996;347(8997):281-4.

23.Phillips V, Graham CT, Manek S, Mccluggage WGJJoCP. The effects of the levonorgestrel intrauterine system (Mirena coil) on endometrial morphology. 2003;56(4):305-7.

24.Hejmadi RK, Chaudhri S, Ganesan R, Rollason TPJIJoSP. Morphologic changes in the endometrium associated with the use of the mirena coil: a retrospective study of 106 cases. 2007;15(2):148-54.

25.Hidalgo MM, Hidalgoregina C, Bahamondes MV, Monteiro I, Petta CA, Bahamondes LJC. Serum levonorgestrel levels and endometrial thickness during extended use of the levonorgestrel-releasing intrauterine system. 2009;80(1):84-9.

26.Hidalgo M, Bahamondes L, Perrotti M, Diaz J, Dantas-Monteiro C, Petta C. Bleeding patterns and clinical performance of the levonorgestrel-releasing intrauterine system (Mirena) up to two years1 1Mirena is a registered trademark of Leiras Oy, Turku, Finland. Contraception. 2002;65(2):129-32.

27.Jensen JT, Mansour D, Lukkarilax E, Inki P, Burock K, Fraser ISJC. Bleeding patterns with the levonorgestrel-releasing intrauterine system when used for heavy menstrual bleeding in women without structural pelvic pathology: a pooled analysis of randomized controlled studies . 2013;87(1):107-12.

28.Chang Y, Tsai EM, Long CY, Lee CL, Kay N. Resectoscopic treatment combined with sonohysterographic evaluation of women with postmenstrual bleeding as a result of previous cesarean delivery scar defects. American Journal of Obstetrics and Gynecology. 2009;200(4):370.e1-.e4.

29.Feng Y-L, Li M-X, Liang X-q, Li X-M. Hysteroscopic Treatment of Postcesarean Scar Defect. Journal of Minimally Invasive Gynecology. 2012;19(4):498-502.

30.Li C, Guo Y, Liu Y, Cheng J, Zhang WJJoPM. Hysteroscopic and laparoscopic management of uterine defects on previous cesarean delivery scars. 2014;42(3):363-70.

Figure legends

Figure 1. Flow chart

\section{Supporting information}

Additional supporting information may be found in the online version of this article:

Figure S1. Intraoperative images of niche characteristics during hysteroscopic correction surgery.

Cyst-like formations B. polyp-like structures C. lateral branch D. abnormal blood vessels

Figure S2. Bleeding/spotting Diary

Patients completed a menstrual diary to record the intensity of bleeding/spotting based on her subjective impression of the heaviest flow for that day.

*vaginal bleeding/spotting; 1-5: amount of bleeding;

1: spotting, 2: light flow bleeding; 3-4:normal flow bleeding; 5: heavy flow bleeding.

Figure S3. Measurement procedure of niche in MRI images.

W: width L: length D: depth TRM: thickness of residual myometrium

Figure S4. efficacy change

S4-A. The effective rate of postmenstrual spotting based on intention to treat analyses within 1 year;

S4-B. The effective rate of postmenstrual spotting based on per protocol analyses within 1 year; 
LNG-IUD = Levonorgestrel - releasing intrauterine device;

$\mathrm{P}$ for trend was calculated by the Linear-by-linear association test;

The shorten time of spotting days $>2$ days was defined as effective case;

Effective case was defined as patient with reduction of spotting $>2$ days;

Effective rate $=$ number of effective cases / number of follow-up cases at each follow-up point.

Table S1. Comparison of improvement of abnormal uterine bleeding based on per protocol analyses

Table S2. Complications between LNG-IUD and hysterocopy group within 1 year

\section{Hosted file}

Figure 1. flow chart.docx available at https://authorea.com/users/356413/articles/479270the-effect-of-a-hysteroscopic-niche-resection-compared-with-lng-iud-on-postmenstrualspotting-in-women-with-a-symptomatic-niche-in-the-uterine-cesarean-scar-a-prospectivecohort-study

\section{Hosted file}

table 1.docx available at https://authorea.com/users/356413/articles/479270-the-effect-of-ahysteroscopic-niche-resection-compared-with-lng-iud-on-postmenstrual-spotting-in-womenwith-a-symptomatic-niche-in-the-uterine-cesarean-scar-a-prospective-cohort-study

\section{Hosted file}

table 2.docx available at https://authorea.com/users/356413/articles/479270-the-effect-of-ahysteroscopic-niche-resection-compared-with-lng-iud-on-postmenstrual-spotting-in-womenwith-a-symptomatic-niche-in-the-uterine-cesarean-scar-a-prospective-cohort-study

\section{Hosted file}

table 3.docx available at https://authorea.com/users/356413/articles/479270-the-effect-of-ahysteroscopic-niche-resection-compared-with-lng-iud-on-postmenstrual-spotting-in-womenwith-a-symptomatic-niche-in-the-uterine-cesarean-scar-a-prospective-cohort-study 